\title{
Cooperative Photoredox and Asymmetric Catalysis
}

\author{
Haohua Huo and Eric Meggers ${ }^{\star a b}$
}

\begin{abstract}
Chemical processes combining visible-light-activated redox catalysis with asymmetric catalysis are reviewed, including enamine catalysis in the presence or absence of an additional photoredox sensitizer, phase transfer catalysis exploiting an in situ-generated electron donor-acceptor complex, photosensitized chiral Lewis acid catalysis, and photoactivated nickel-catalyzed asymmetric cross-couplings. The transfer of a single electron leads to intermediate radical ions, whose strongly modulated reactivities can be exploited for asymmetric catalysis in a novel fashion. All processes discussed here are redox neutral so that the electron serves as a real catalyst which cooperates with an asymmetric catalyst for the overall asymmetric transformation.
\end{abstract}

Keywords: Asymmetric catalysis - Electron transfer - Electron transfer catalysis - Photoredox catalysis . Visible light

\section{Introduction}

The investigation and design of organic reaction mechanisms which involve single electron transfer (SET) steps have witnessed a remarkable renaissance over the last several years. ${ }^{11]}$ What makes such reactions so attractive are the dramatic effects that a single electron more or less can cause. Starting from a closed shell molecule, the withdrawal or addition of a single charge generates intermediate radical ions with strongly modulated reactivities. ${ }^{[2]}$ For example, removing a single electron from toluene dramatically decreases its $\mathrm{p} K_{\mathrm{a}}$ value $\left(\Delta \mathrm{p} K_{\mathrm{a}}=63\right.$ in DMSO), thereby converting it into a strong acid. ${ }^{[3]}$ Clearly, radical ion chemistry provides untapped opportunities to develop novel and synthetically useful chemical transformations.

Under the conditions that the reaction is overall redox neutral and the mechanism follows the order electron transfer - chemical $\operatorname{step}(\mathrm{s})$ - electron transfer, the electron constitutes a catalyst. ${ }^{[4,5]}$ Such reactions were previously frequently termed 'elec-

${ }^{*}$ Correspondence: Prof. Dr. E. Meggers

aFachbereich Chemie, Philipps-Universität Marburg Hans-Meerwein-Strasse 4, 35043 Marburg, Germany ${ }^{\mathrm{b}}$ College of Chemistry and Chemical Engineering Xiamen University, Xiamen 361005, P. R. China E-mail: meggers@chemie.uni-marburg.de tron transfer catalysis' and are nowadays often called 'redox catalysis' or 'photoredox catalysis' if the redox chemistry is activated by light. ${ }^{[6]}$ However, as a cautionary note, in the case of 'photoredox catalysis', the aspect of catalysis sometimes refers to the catalytic activity of the photoredox sensitizer, not to the overall redox process. Studer and Curran recently suggested the term 'electron catalysis' for related chain processes since the electron indeed serves formally as a real catalyst, in analogy to the proton serving as the formal catalyst in Brønsted acid catalyzed reactions. ${ }^{[5]}$

For electron-(transfer)-catalyzed reactions, photoinduced electron transfer (PET), by means of using catalytic amounts of a light-activated photosensitizer, probably constitutes the mildest and often most favorable method to initiate the transfer of a single electron. ${ }^{[7]}$ Upon the absorption of light, the photosensitizer occupies an electronically excited state which is a superior electron donor as well as acceptor compared to its ground state. What makes the photosensitization scheme so useful is the fact that it allows the production of very low steady state concentrations of a strong oxidant or strong reducing agent, which is a key advantage over chemical oxidants or electrochemistry, since reactions through radical ions often involve intermediates which are more prone to further oxidation or reduction compared to the initial substrates. Whereas photoinduced electron transfer was in the early days mainly based on UV-light activation, ${ }^{[7]}$ the recent renewed boom of photochemistry stems to a significant degree from improved photosensitizers that allow an activation with visible light, which in form of solar light constitutes an environmentally friendly and sustainable source of energy for de- livering the activation energy of chemical reactions. ${ }^{[8]}$ Furthermore, photochemistry with visible-light-activated photosensitizers does not need any specialized equipment and is nowadays typically performed with energy light saving lamps (compact fluorescent lamps, CFL) or LED light sources.

In this review, we will focus on examples in which photoredox catalysis, in the strict sense of photoactivated single electron transfer catalysis, cooperates with asymmetric catalysis. Asymmetric catalysis holds promise as one of the most economical strategies for the synthesis of non-racemic compounds. The last several years have revealed that electron-transferinduced reactions through radical ions allow the design of catalytic, asymmetric reactions with novel mechanisms. However, at the same time, the high reactivities and limited lifetimes of radical ion and radical intermediates pose a significant challenge to interface them with asymmetric catalysis, indicated by the still limited number of mechanistically distinct electroncatalyzed, catalytic asymmetric reaction schemes. This scholarly review discusses the most important strategies in which photoredox catalysis has been successfully merged with asymmetric catalysis. ${ }^{[9]}$

\section{Enamine Catalysis}

In a seminal publication from 2008 , MacMillan introduced a strategy to merge visible-light-activated photoredox catalysis with asymmetric organocatalysis to realize the challenging direct enantioselective $\alpha$-alkylation of aldehydes. ${ }^{[10]}$ This pioneering work spurred a lot of excitement since it provided a terrific 
blueprint for how to merge radical ion chemistry with asymmetric organocatalysis in an elegant fashion, and subsequent work by MacMillan and others demonstrated the generality of this strategy. ${ }^{[11-18]}$ According to the detailed mechanism shown in Scheme 1, an enantioselective intermolecular $\alpha$-alkylation of aldehydes with acceptor-substituted alkyl halides was accomplished with a combination of a transition metal photosensitizer and an imidazolidinone organocatalyst $\mathbf{1}$. Work by Zeitler, König, Pericàs, and others later demonstrated that organic dyes, inorganic semiconductors, and other transition metal complexes are also suitable photosensitizers in this and related systems. ${ }^{[13-15,18]}$ The catalytic cycle is initiated by the transfer of a single electron to an electrophile, such as acceptor-substituted benzyl bromides, phenacyl bromides, perfluoroalkyl iodides, or $\alpha$-bromonitriles followed by the release of a halide or pseudohalide leaving group with the formation of a carbon-centered radical. This electron-deficient radical rapidly reacts with the electron-rich double bond of the intermediate enamine, formed by condensation of the aldehyde substrate with the secondary amine catalyst, in a stereocontrolled fashion. Importantly, to realize catalysis with respect to electron transfer, the hereby generated $\alpha$-aminoalkyl radical is very prone to oxidation and upon removal of an electron, the formed iminium ion hydrolyzes and releases the chiral amine for another enamine catalysis cycle. Thus, the overall reaction is redox neutral: the single electron reduction of the electrophile is followed by a single electron oxidation of the intermediate $\alpha$-aminoalkyl radical. Quantum yields determined by Yoon and coworkers for a representative model reaction reveal that this intermediate $\alpha$-aminoalkyl radical can transfer the electron directly to another electrophile substrate, thereby rendering this reaction a chain process. ${ }^{[19]}$ Once a termination of the chain process occurs, the light-activated photosensitizer initiates a new chain process. It can be expected that the chain length will vary significantly with the nature of the used electrophile. In general, the involvement of a chain reaction is highly desirable because it allows the light energy to be utilized more efficiently.

With respect to controlling the reaction path and the stereoselectivity of the highly reactive intermediate carbon-based radicals, the success of this asymmetric catalysis is based on the key aspect that the secondary amine catalyst ${ }^{[20]}$ converts the aldehyde substrate into an intermediate enamine featuring an electron-rich $\pi$-bond that is highly reactive towards electron-deficient radicals. This circumvents potential side reactions and also permits control of the stereochemistry of the radical addi-

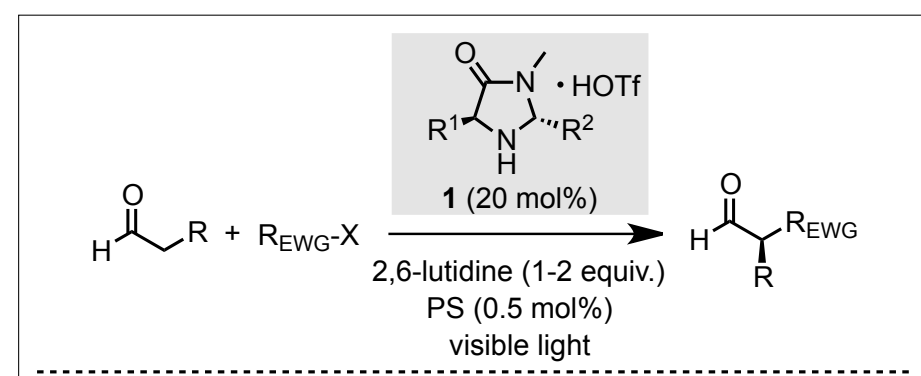

Examples:

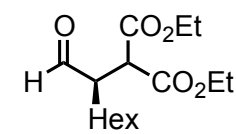<smiles>[14CH3]C(C=O)C(F)(F)F</smiles><smiles>[R][C@H](C=O)CC(=O)c1ccccc1</smiles>

$93 \%$ yield, $90 \%$ ee $79 \%$ yield, $99 \%$ ee $84 \%$ yield, $96 \%$ ee

Putative mechanism:

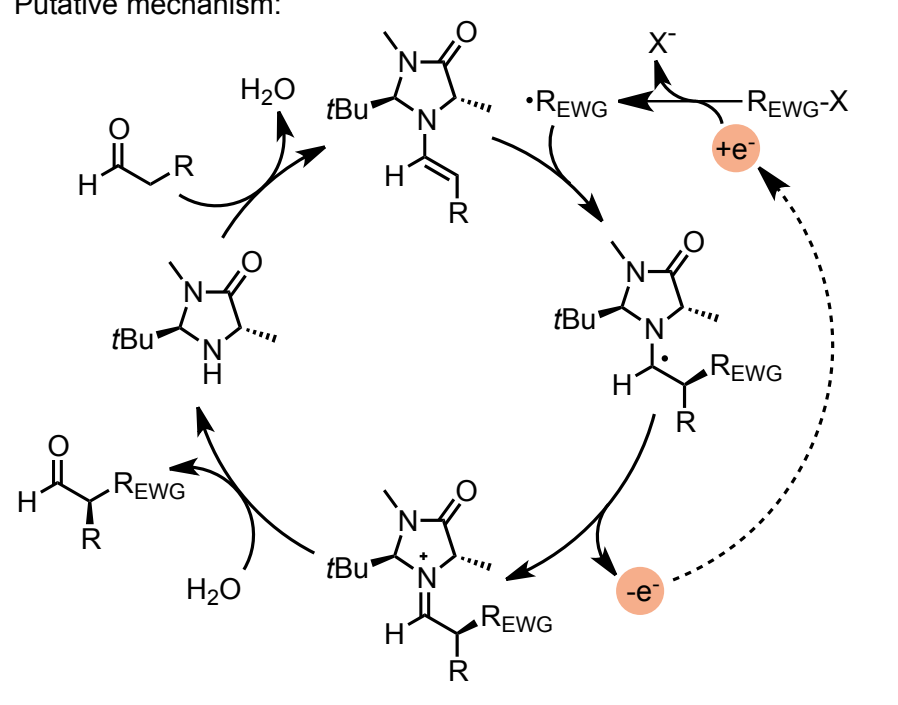

Scheme 1. Enantioselective $\alpha$-alkylation of aldehydes via merger of organocatalysis and photoredox catalysis. $R_{E W G}=$ acceptor-substituted benzyl bromides, phenacyl bromides, diethyl bromomalonate perfluoroalkyl iodides, and $\alpha$-bromonitriles. PS = visible light photosensitizer in the form of $\left[R u(b p y)_{3}\right]$ $\mathrm{Cl}_{2}$, [Ir(ppy) $\left.)_{2}(\mathrm{dtbbpy})\right]$ $\mathrm{PF}_{6}$, fac-[Ir(ppy) $)_{3}$, $\left[\mathrm{Fe}(\mathrm{bpy})_{3}\right] \mathrm{Br}_{2}$, eosin $\mathrm{Y}$, or inorganic semiconductors. tion through the chirality of the secondary amine catalyst.

In a related strategy to generate radicals through reductive fragmentation and interface them with enamine catalysis, MacMillan introduced a visible-lightinduced enantioselective $\alpha$-amination of aldehydes using 2,4-dinitrophenylsulfonyloxy- $N$-functionalized carbamates and chiral secondary amine $\mathbf{2}$ as the catalyst (Scheme 2).[21] A single electron transfer to dinitrophenylsulfonyloxy- $N$-functionalized carbamates leads to the release of 2,4-dinitrophenylsulfonate with the concomitant formation of an (electrondeficient) $N$-centered radical which subsequently undergoes a radical addition to the electron-rich $\pi$-bond of a chiral enamine intermediate. In analogy to the previous mechanism (Scheme 1), it is likely that the herewith formed $\alpha$-aminoalkyl radical directly reduces another substrate dinitrophenylsulfonyloxy- $N$-functionalized carbamate, thereby generating a chain process. A long chain length can be expected for this reaction due to a probably inefficient photoinitiation of this process and the lack of an additional photosensitizer.

In the context of photoactivat- ed asym,metric enamine chemistry, Melchiorre and coworkers recently revealed that the photoactivated asymmetric $\alpha$-alkylation of aldehydes and ketones with electron-deficient benzyl bromides and phenacyl bromides can even be executed in the absence of an external photoredox sensitizer (Scheme 3). ${ }^{[22,23]}$ Mechanistic work revealed that a colored charge transfer (electron donor-acceptor, EDA) complex 3 composed of the in situ formed electronrich enamine and electron-deficient acceptor-substituted organobromide absorbs visible light (as opposed to the individual components) and induces a single electron transfer from the enamine to the organobromide. Thus, EDA formation allows SETtriggered redox neutral transformations to be performed without the involvement of external photosensitizers. In a recent twist of this story, Melchiorre reported that even in the absence of any EDA complex, enamines are capable of absorbing sufficient visible light to induce an electron transfer from an electronic excited state of the enamine (4) to bromomalonates, thereby triggering a chain process with involvement of the enamine in the ground state, in analogy to the mechanism depicted in Scheme 1.[24] 

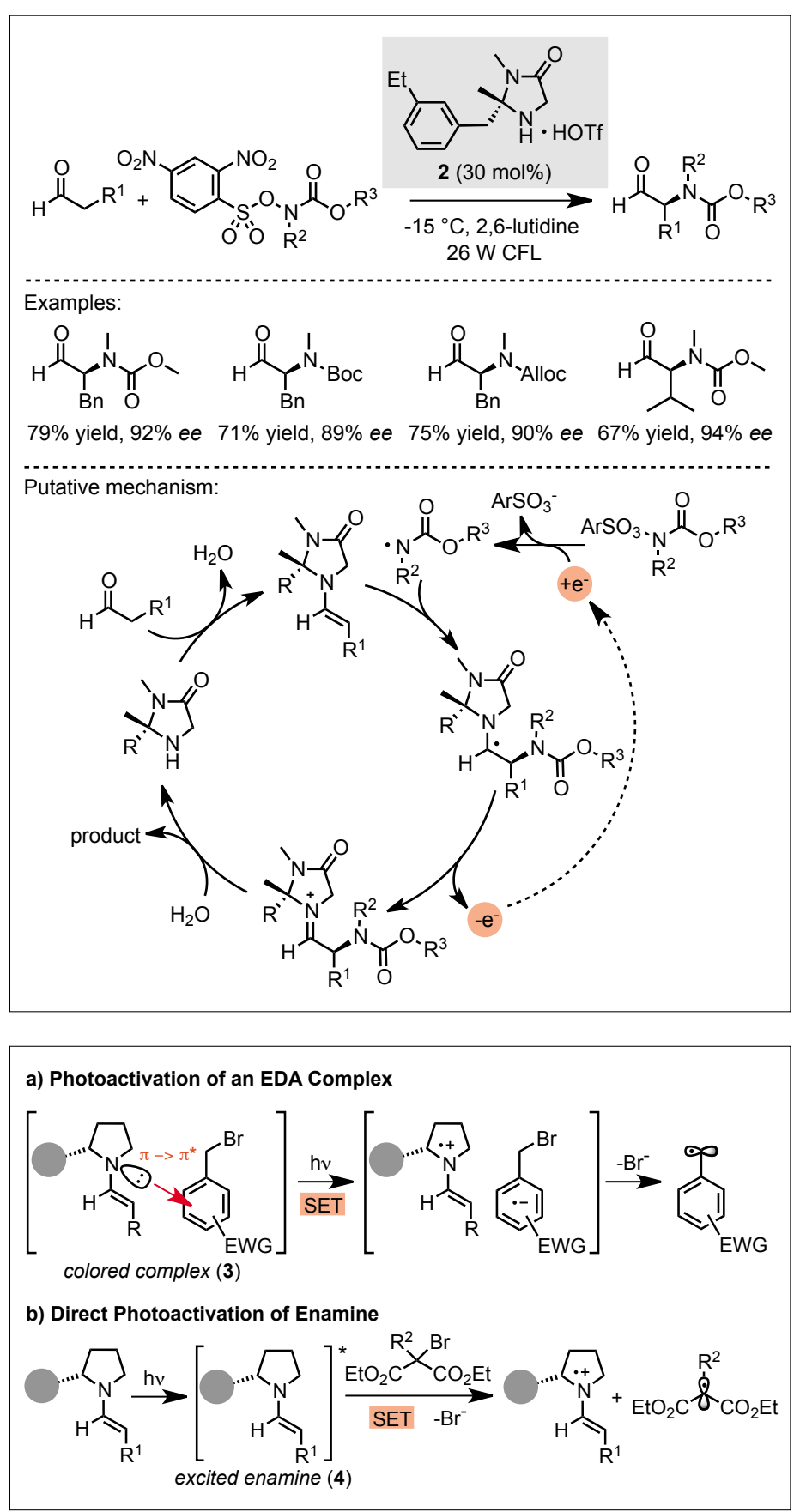

Scheme 2.

Asymmetric $\alpha$-amination of aldehydes via photoinduced electron catalysis.

Scheme 3.

Mechanisms for the involvement of enamines in the photochemical generation of radicals.

\section{Phase Transfer Catalysis}

Melchiorre recently further advanced the photoactivation of EDA complexes to a photochemical enantioselective perfluoroalkylation of $\beta$-ketoesters using phase-transfer-catalyst (PTC) 5 (Scheme 4). ${ }^{[25]}$ The proposed radical chain reaction is initiated by the photochemical activation of an EDA complex out of enolate (coordinated to the cationic PTC) and the perfluoroalkyl iodide $\left(\mathrm{R}_{\mathrm{F}}-\mathrm{I}\right)$ through $\pi-\sigma^{*}$ interactions. A visible-light promoted electron transfer within the EDA from the enolate to $\mathrm{R}_{\mathrm{F}}-\mathrm{I}$ leads to a reductive cleavage of the $\mathrm{C}-\mathrm{I}$ bond and the thereby generated electrophilic perfluoroalkyl radical can next react with the electron-rich $\pi$-bond of (another) enolate to provide an intermediate ketyl. The stereoselectivity of this step is controlled by the chiral PTC counterion. Finally, the intermediate ketyl converts to the product either by directly participating in an SET with another $\mathrm{R}_{\mathrm{F}}-\mathrm{I}$, or alternatively by abstracting an iodine from $\mathrm{R}_{\mathrm{F}}-\mathrm{I}$, followed by iodide dissociation. Overall, this together with previous work from the Melchiorre laboratory demonstrates the versatility of the photochemical activation of EDA complexes for devising visiblelight-driven transformations ("photo-organocatalysis') without the need for any additional external photosensitizer.

\section{Lewis Acid Catalysis}

Yoon recently reported an electroncatalyzed [2+2]-cycloaddition that is interfaced with asymmetric chiral Lewis acid catalysis by using Eu(OTf) $(10 \mathrm{~mol} \%)$ in combination with a chiral coordinating ligand (20-30 mol\%) and $\left[\mathrm{Ru}(\mathrm{bpy})_{3}\right]^{2+}$ as the photoredox sensitizer (Scheme 5). [26] The proposed mechanism starts with the photoactivation of $\left[\mathrm{Ru}(\mathrm{bpy})_{3}\right]^{2+}$, followed by a single electron from $i \mathrm{Pr}_{2} \mathrm{NEt}$ to generate $\left[\operatorname{Ru}(\mathrm{bpy})_{3}\right]^{+}$, which then serves as the reducing agent and transfers a single electron to a Lewis acid coordinated aryl enone, thereby generating an intermediate radical anion. The latter participates in an efficient $[2+2]$-cycloaddition reaction with another Michael acceptor. The formed cyclobutane containing ketyl radical subsequently loses an electron and thereby closes the electron catalysis cycle. Apparently, all reactive intermediates remain coordinated to the chiral europium(III) Lewis acid throughout the catalytic cycles so that high enantio- and diastereoselectivities can be reached. The authors mention that no background reaction occurs in the absence of the Lewis acid, which contributes to the high enantioselectivities. Furthermore, the authors demonstrate that converting the Schiff base ligand 6 into the corresponding reduced secondary amine 7 effects a complete switch in diastereoselectivity from 1,2-trans to 1,2-cis cyclobutanes, which means that the chiral ligand can control both the relative and absolute stereochemistry of the [2+2]-photocycloaddition products.

In another example of cooperative photoredox and asymmetric Lewis acid catalysis, the Yoon group reported an enantioselective addition of $\alpha$-aminomethyl radicals to $\alpha, \beta$-unsaturated carbonyl compounds (Scheme 6).[27] Built on insights by Mariano ${ }^{[28]}$ and Pandey ${ }^{[29]}$ that $\alpha$-silylamines undergo a facile oxidative desilylation to generate nucleophilic $\alpha$-amino radicals, which then in turn can add to acceptor-substituted alkenes, Yoon used scandium(III) triflate together with the pybox ligand $\mathbf{8}$ as a chiral Lewis acid in combination with the visible-light-activated photoredox sensitizer $\left[\mathrm{Ru}(\mathrm{bpy})_{3}\right]^{2+}$ to elegantly convert this aminoalkylation into a photoredox-catalyzed assymetric reaction. An optimization study revealed that the addition of $\mathrm{Bu}_{4} \mathrm{NCl}$ was beneficial and that Sibi's pyrazolidinone auxiliary ( $\mathrm{Z}$ in Scheme 6) ${ }^{[30]}$ provided the best results. Mechanistically, it can be expected that the scandium(III) Lewis acid activates the Michael acceptor by bidentate coordination, thereby increasing its reactivity towards the stereocontrolled addition of the nucleophilic $\alpha$-amino radical. A subsequent single electron reduction of the formed $\alpha$-carbonyl radical followed by protonation provides the product and after decomplexation allows another catalytic cycle. Note that this reaction is overall redox neutral and it is likely that some degree 

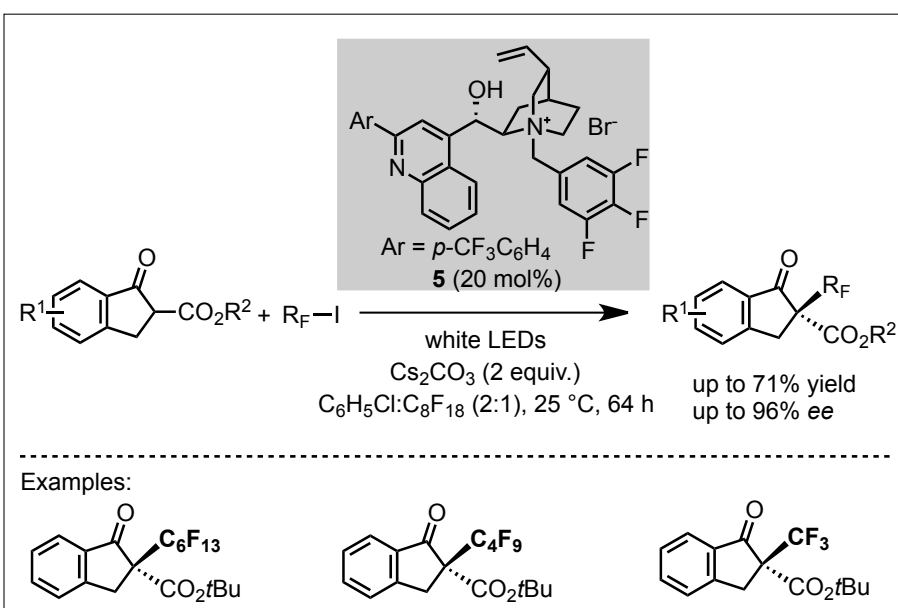

$71 \%$ yield, $93 \%$ ee

$62 \%$ yield, $90 \%$ ee

$50 \%$ yield, $96 \%$ ee

Putative mechanism:

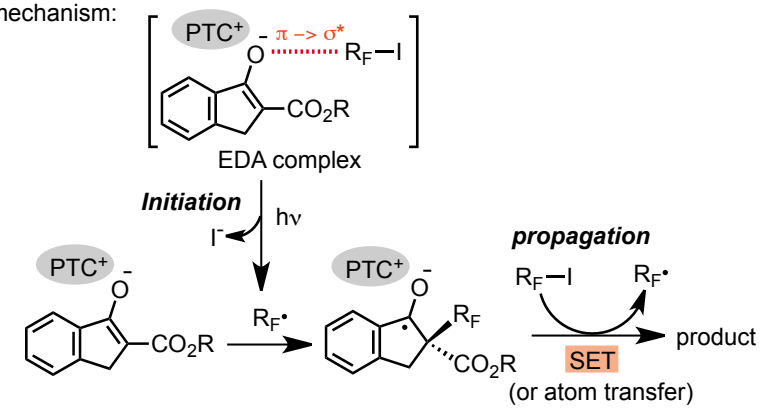

Scheme 4. Visible-light-activated phase-transfer-catalyzed perfluoroalkylation of $\beta$-ketoesters.

of chain reaction is involved as indicated by the dotted arrow in Scheme 6 .

Our laboratory recently reported visible-light-induced asymmetric photoredox catalysis using a single chiral iridium complex which serves a dual function, namely as a catalytically active chiral Lewis acid and at the same time as a precursor for an in situ assembled visible-light-activated photoredox catalyst. ${ }^{[31-33]}$ In the iridium complex 9, the octahedral metal center is cyclometalated with two bidentate ligands in a propeller-type fashion. Two additional acetonitriles are exchange-labile and allow substrates to become activated by twopoint binding. This complex is structurally very simple and only contains achiral ligands, with metal-centered chirality (metal centrochirality) being the exclusive source of chirality. Importantly, despite the two labile acetonitrile ligands, the catalyst is configurationally inert and retains its relative and absolute configuration throughout the catalysis. ${ }^{[34]}$ Iridium complex 9 is a highly effective asymmetric photoredox catalyst for the visible-light-induced enantioselective $\alpha$-alkylation of 2-acyl imidazoles with electron-deficient benzyl bromides $^{[31]}$ and phenacyl bromides ${ }^{[31]}$ as well as $\alpha$-trichloromethylation ${ }^{[32]}$ of 2-acyl imidazoles and 2-acylpyridines.

Our proposed mechanism is shown in Scheme 7, which can be classified as an

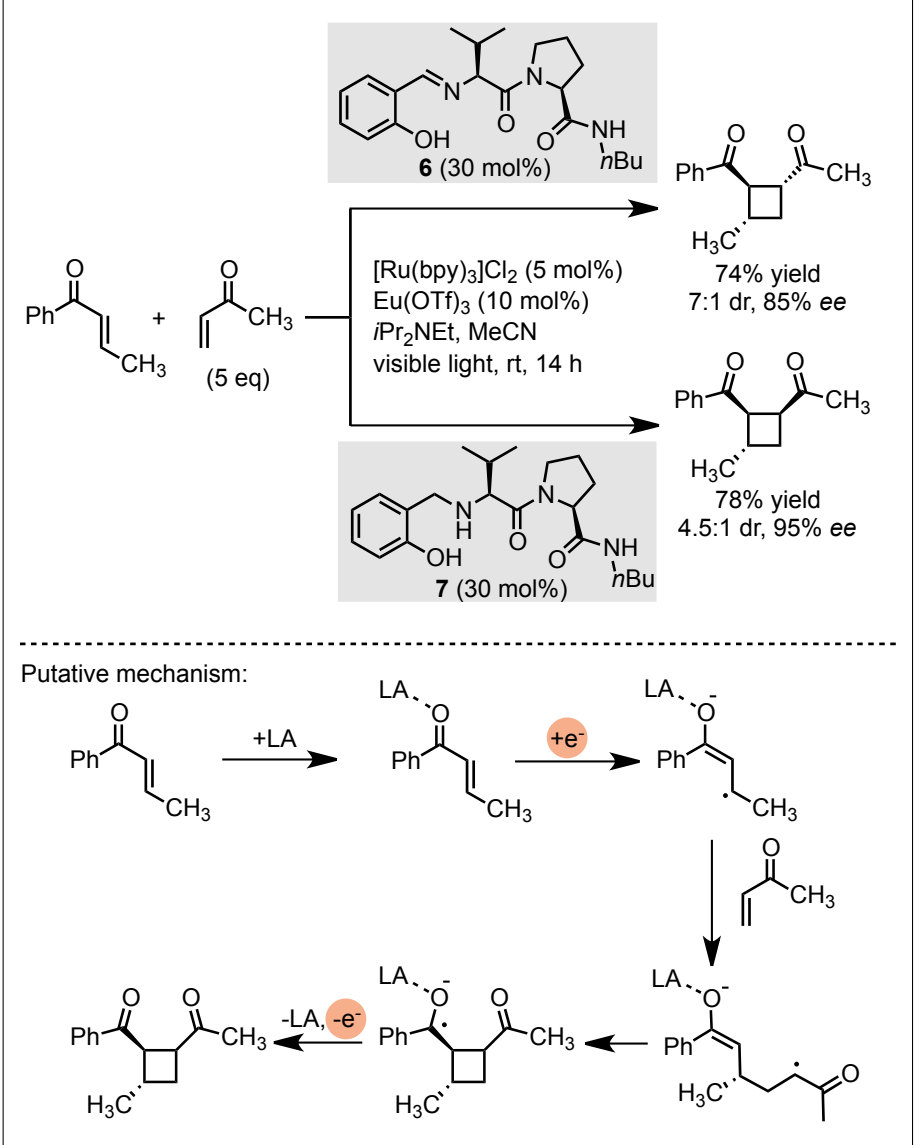

Scheme 5. Asymmetric Lewis acid catalyzed [2+2]-cycloaddition of $\alpha, \beta-$ unsaturated ketones via photoredox catalysis. $L A=$ chiral Lewis acid catalyst.

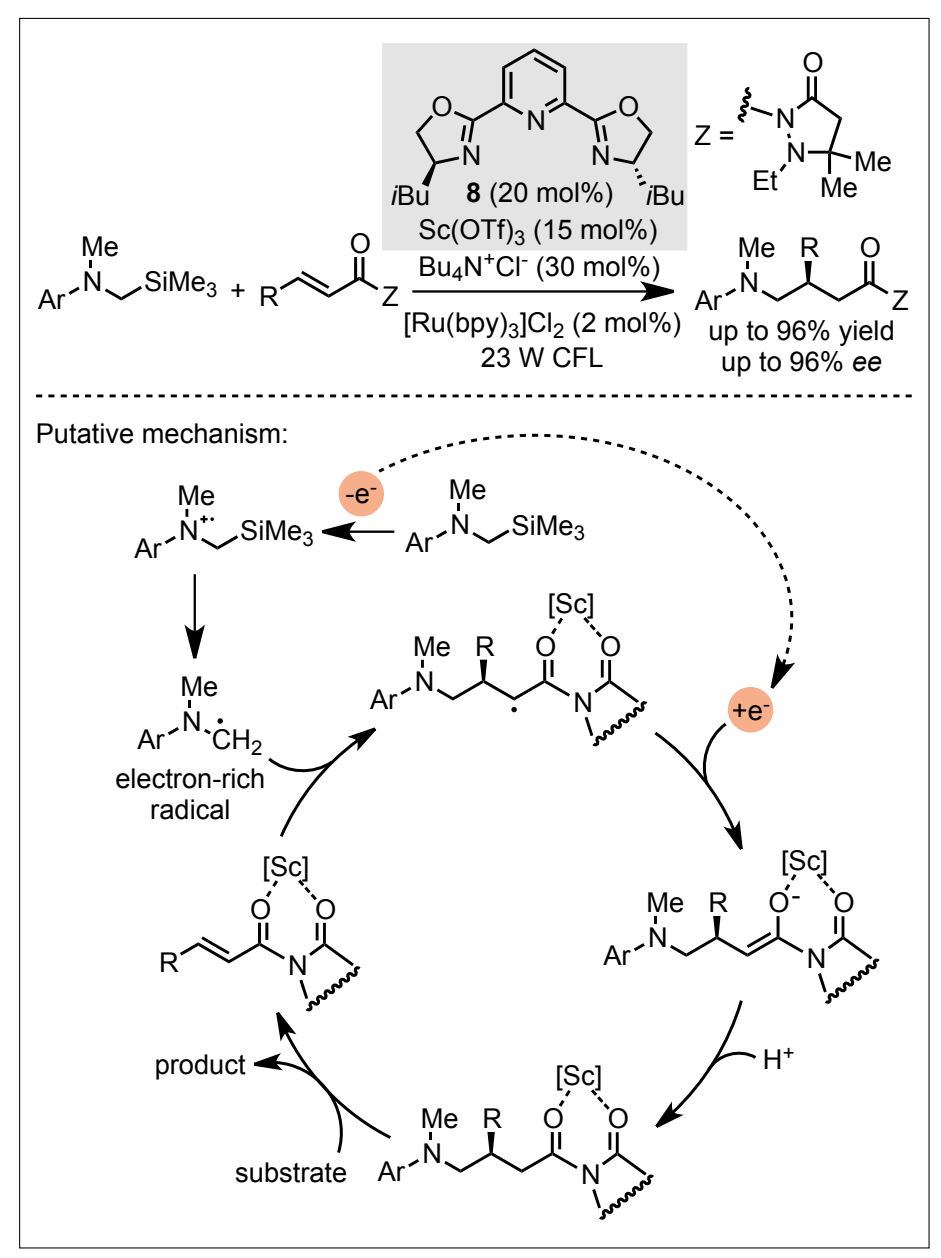

Scheme 6. Dual Lewis acid/photoredox catalysis system for the enantioselective aminoalkylation of $\alpha, \beta$-unsaturated carbonyl compounds. 
electron-transfer-catalyzed nucleophilic substitution via $\mathrm{S}_{\mathrm{RN}} 1$. Accordingly, the catalytic cycle is initiated by bidentate coordinating of the 2-acylimidazole or 2-acylpyridine substrate to the iridium catalyst (intermediate I), followed by base-promoted deprotonation to an electron-rich iridium enolate (intermediate II). The subsequent addition of a reductively generated electrophilic carbon-centered radical to the nucleophilic enolate double bond provides an iridium-coordinated ketyl radical (intermediate III), which is oxidized to an iridium-coordinated product (intermediate IV), followed by product release. The described catalytic cycle intertwines with a photoredox cycle that generates the electrophilic carbon radical upon SET from the photoactivated photosensitizer to the organobromide substrate and subsequent release of bromide. Determined quantum yields of $>1$ for the trichlormethylation reveal that the electrophilic radical is also formed by direct electron transfer from the strongly reducing ketyl radical intermediate III to the electron-deficient organobromide, thereby leading to a chain propagation. A number of mechanistic experiments suggest that it is the neutral intermediate iridium enolate complex (II) that serves as the active photosensitizer. Thus, the chiral iridium enolate II is a key intermediate, providing an excellent asymmetric induction in the reaction with electrophilic radicals and simultaneously serves as the visible-light-activated photosensitizer for triggering electron catalysis.

\section{Nickel Catalysis}

Molander ${ }^{[35]}$ and Doyle with MacMillan[36] recently reported an intriguing merger of photoredox catalysis and nickel-catalyzed cross-coupling. In the case of the Molander system, alkoxyalkyl- and benzyl trifluoroborates were cross-coupled with aryl bromides under very mild conditions using a nickel catalyst and a photosensitizer in combination with visible light. ${ }^{[35,37-39]}$ Mechanistically, the single electron oxidation of the trifluoroborate induces a fragmentation into $\mathrm{BF}_{3}$ and an alkylradical which is supposed to add to nickel(0) and leads to the formation of an alkylnickel(I) intermediate, which in turn undergoes an oxidative addition with the aryl bromide to form a pentacoordinate nickel(III) intermediate. Alternatively, the oxidative addition can precede the radical combination with nickel. The intermediate nickel(III) complex undergoes a rapid reductive elimination under release of the cross-coupling product and a nickel(I) species which then takes up an electron to close the catalytic cycle. As indicated in Scheme 8 , the overall process is redox neutral by

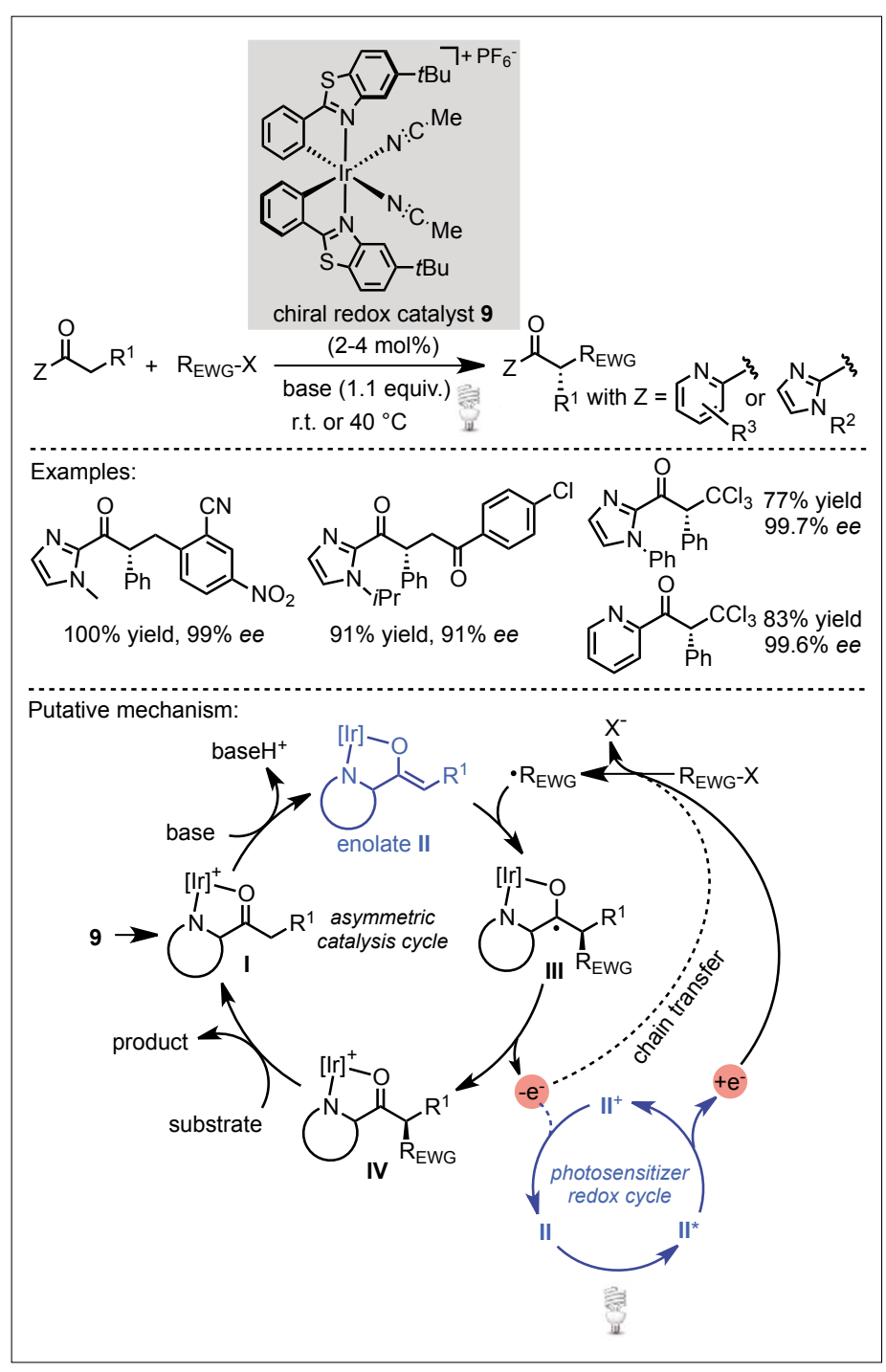

Scheme 7. Proposed mechanism for combining photoredox and asymmetric catalysis with a chiral iridium complex. Iridium enolate complex II constitutes the in situ formed photosensitizer and serves as the key intermediate in the asymmetric cycle $(\mathrm{EWG}=$ electron withdrawing group). including one oxidative and one reductive single electron transfer step. Interestingly, by employing a chiral bisoxazoline ligand, this process can be rendered enantioselective, albeit with currently only modest enantioselectivities. ${ }^{[35,38]}$

\section{Conclusions}

Electron-catalyzed reactions open intriguing new options for devising chemical reactions. With respect to electroncatalyzed asymmetric catalysis, to date, only a limited number of successful processes have been reported including lightactivated enamine, phase transfer, chiral Lewis acid, and nickel catalysis. ${ }^{[40]}$ The challenge remains to tame and control the reactivity of the intermediate radical species and to interface them with asymmetric catalysis cycles. This requires either a strong activation of a substrate towards the reaction with a radical species or the generation of the radical species while attached to the catalyst. In this respect, the use of chiral transition metal complexes which can both serve as the photosensitizer and asymmetric catalyst might allow other reaction schemes in which photoactivation and reactivity are intertwined in unique ways. Finally, it has to be mentioned that photoinduced electron transfer processes can be complicated by the kinetics of the involved electron transfer processes (such as the energy-wasting back electron transfer). Regardless, the authors are looking forward to new innovative strategies for achieving cooperative asymmetric photoredox catalysis.

Received: October 6, 2015 


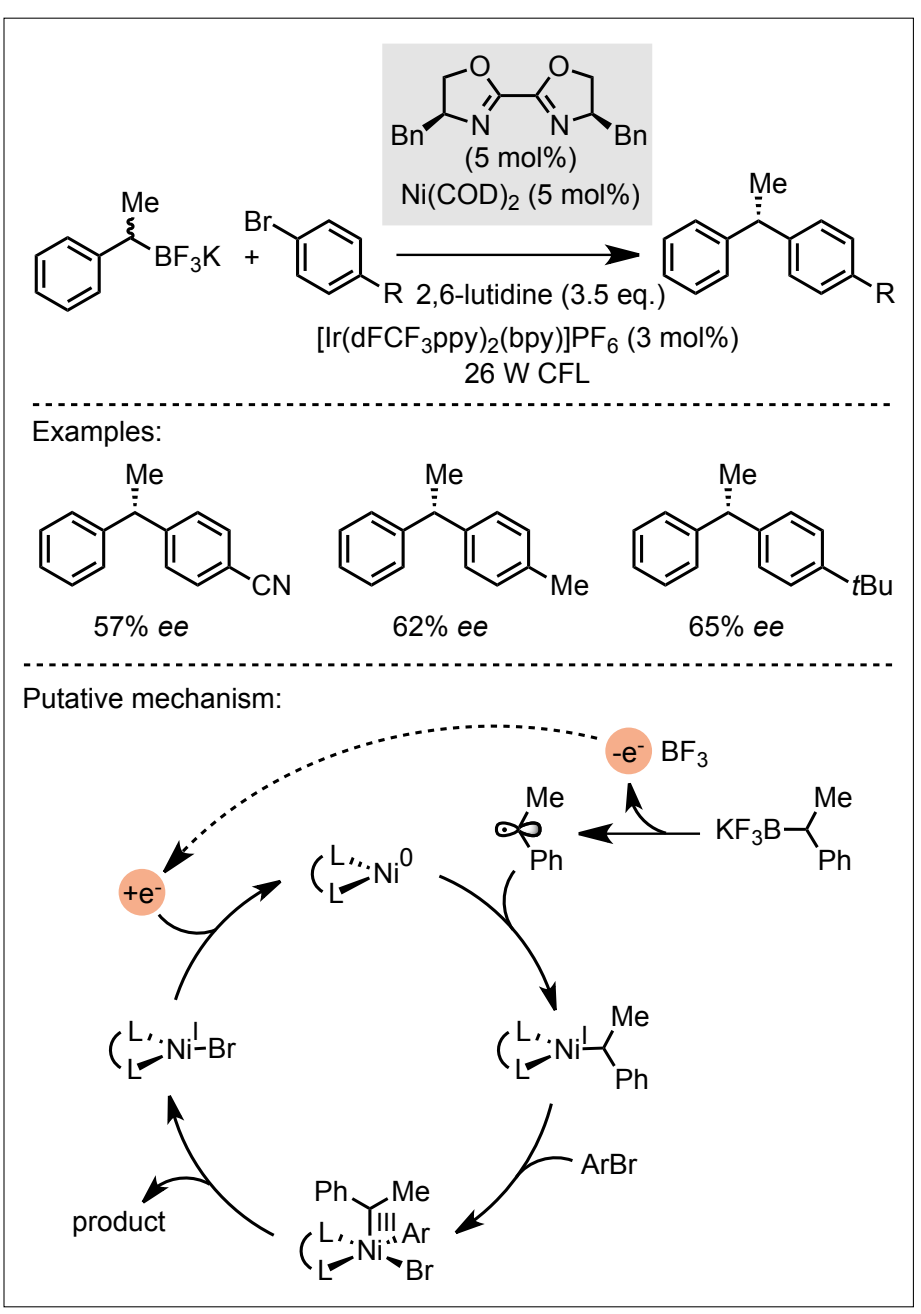

[1] Reactions involving single electron transfer: N. Zhang, S. R. Samanta, B. M. Rosen, V. Percec, Chem. Rev. 2014, 114, 5848.

[2] a) M. Schmittel, A. Burghart, Angew. Chem. Int Ed. Engl. 1997, 36, 2550; b) M. A. Ischay, T. P. Yoon, Eur. J. Org. Chem. 2012, 3359.

[3] F. G. Bordwell, J. P. Cheng, J. Am. Chem. Soc. 1989, 111, 1792.

[4] M. Chanon, M. L. Tobe, Angew. Chem. Int. Ed. Engl. 1982, 21, 1.

[5] A. Studer, D. P. Curran, Nature Chem. 2014, 6, 765.

[6] Photoredox catalysis: a) M. Reckenthäler, A. G. Griesbeck, Adv. Synth. Catal. 2013, 355, 2727; b) C. K. Prier, D. A. Rankic, D. W. C. MacMillan, Chem. Rev. 2013, 113, 5322.

[7] Photoinduced electron transfer: a) J. Mattay, Angew. Chem. Int. Ed. Engl. 1987, 26, 825; b) M. Fagnoni, D. Dondi, D. Ravelli, A. Albini, Chem. Rev. 2007, 107, 2725.
Scheme 8. Nickelcatalyzed stereoconvergent crosscoupling.
[14] M. Cherevatskaya, M. Neumann, S. Füldner, C. Harlander, S. Kümmel, S. Dankesreiter, A. Pfitzner, K. Zeitler, B. König, Angew. Chem. Int. Ed. 2012, 51, 4062

[15] P. Riente, A. Matas Adams, J. Albero, E. Palomares, M. A. Pericàs, Angew. Chem. Int. Ed. 2014, 53, 9613.

[16] Y. Zhu, L. Zhang, S. Luo, J. Am. Chem. Soc. 2014, 136, 14642 .

[17] E. R. Welin, A. A. Warkentin, J. C. Conrad, D. W. C. MacMillan, Angew. Chem. Int. Ed. 2015, $54,9668$.

[18] A. Gualandi, M. Marchini, L. Mengozzi, M. Natali, M. Lucarini, P. Ceroni, P. G. Cozzi, ACS Catal. 2015, 5, 5927.

[19] M. A. Cismesia, T. P. Yoon, Chem. Sci. 2015, 6, 5426.

[20] S. Mukherjee, J. W. Yang, S. Hoffmann, B. List, Chem. Rev. 2007, 107, 5471.

[21] G. Cecere, C. M. König, J. L. Alleva, D. W. C. MacMillan, J. Am. Chem. Soc. 2013, 135, 11521.

[22] E. Arceo, I. D. Jurberg, A. Álvarez-Fernández, P. Melchiorre, Nature Chem. 2013, 5, 750.

[23] E. Arceo, A. Bahamonde, G. Bergonzini, P. Melchiorre, Chem. Sci. 2014, 5, 2438.

[24] M. Silvi, E. Arceo, I. D. Jurberg, C. Cassani, P. Melchiorre, J. Am. Chem. Soc. 2015, 137, 6120.

[25] Ł. Woźniak, J. J. Murphy, P. Melchiorre, J. Am. Chem. Soc. 2015, 137, 5678.

[26] J. Du, K. L. Skubi, D. M. Schultz, T. P. Yoon, Science 2014, 344, 392.

[27] L. Ruiz Espelt, I. S. McPherson, E. M. Wiensch, T. P. Yoon, J. Am. Chem. Soc. 2015, 137, 2452.

[28] U. C. Yoon, P. S. Mariano, Acc. Chem. Res. 1992, 25, 233.

[29] G. Pandey, Synlett 1992, 546.

[30] M. P. Sibi, L. Venkatraman, M. Liu, C. P. Jasperse, J. Am. Chem. Soc. 2001, 123, 8444.

[31] H. Huo, X. Shen, C. Wang, L. Zhang, P. Röse, L.-A. Chen, K. Harms, M. Marsch, G. Hilt, E. Meggers, Nature 2014, 515, 100.

[32] H. Huo, C. Wang, K. Harms, E. Meggers, J. Am. Chem. Soc. 2015, 137, 9551.

[33] For related oxidative asymmetric photoredox catalysis, see: C. Wang, Y. Zheng, H. Huo, P. Röse, L. Zhang, K. Harms, G. Hilt, E. Meggers, Chem. Eur. J. 2015, 21, 7355 .

[34] a) H. Huo, C. Fu, K. Harms, E. Meggers, $J$. Am. Chem. Soc. 2014, 136, 2990; b) X. Shen, H. Huo, C. Wang, B. Zhang, K. Harms, E. Meggers, Chem. Eur. J. 2015, 21, 9720.

[35] J. C. Tellis, D. N. Primer, G. A. Molander, Science 2014, 345, 433.

[36] Z. Zuo, D. T. Ahneman, L. Chu, J. A. Terrett, A. G. Doyle, D. W. C. MacMillan, Science 2014, 345, 437.

[37] D. N. Primer, I. Karakaya, J. C. Tellis, G. A. Molander, J. Am. Chem. Soc. 2015, 137, 2195.

[38] O. Gutierrez, J. C. Tellis, D. N. Primer, G. A Molander, M. C. Kozlowski, J. Am. Chem. Soc. 2015, 137, 4896.

[39] I. Karakaya, D. N. Primer, G. A. Molander, Org. Lett. 2015, 17, 3294.

[40] The following relevant new publications could not be discussed: a) D. Uraguchi, N. Kinoshita, T. Kizu, T. Ooi, J. Am. Chem. Soc. 2015, 137, 13768; b) C. Wang, J. Qin, X. Shen, R. Riedel, K. Harms, E. Meggers, Angew. Chem. Int. Ed. 2016, 55, 685; c) Z. Zuo, H. Cong, W. Li, J. Choi, G. C. Fu, D. W. C. MacMillan, J. Am. Chem. Soc. 2016, 138, 1832; d.) Q. M. Kainz, C. D. Matier, A. Bartoszewicz, S. L. Zultanski, J. C. Peters, G. C. Fu, Science 2016, 351, 681 (note added in proof). 